\title{
Quelle importance accordons-nous à la qualité du système de santé?*
}

\author{
Harry Telsera,b \\ a Polynomics; ' Zentrum für Gesundheit, Politik und Ökonomie (Centre pour la santé, la politique et l'économie), Université de Lucerne
}

Cet article a déjà été publié dans la «Vie économique» 3/2017, p. 23-26.

\footnotetext{
1 Cf. "Qualitäts- und Zufriedenheitsmessungen in der Akutsomatik, der Rehabilitation und der Psychiatrie» de l'Association nationale pour le développement de la qualité dans les hôpitaux et les cliniques (ANQ).

2 Interpharma. L'essentie en bref sur le Moniteur de la santé 2016. Bâle; 2016.

3 OCDE. Examens de l'OCDE des systèmes de santéSuisse. Paris; 2006.

4 Telser, H., S. Vaterlaus, P. Zweifel et P. Eugster (2004) Was leistet unser Gesundheitswesen? Zurich.
}

En se focalisant sur les économies, le débat public oublie bien souvent de rappeler les avantages de notre système de santé pour les patients et les assurés. Ceux-ci sont pourtant prêts à dépenser beaucoup pour une meilleure qualité de vie.

Depuis des années, le débat public sur le système de santé se focalise presque exclusivement sur les coûts. Dans les médias, on diagnostique une "explosion des coûts» et on met en garde contre le "choc des primes". Cette focalisation incite de nombreux politiciens à l'activisme et oriente les débats au Parlement principalement sur la réduction des coûts. Depuis l'entrée en vigueur de la loi sur l'assurance-maladie (LAMal), des voix se sont souvent élevées pour réclamer le rationnement des prestations. Par conséquent, l'Office fédéral de la santé publique (OFSP) a récemment restreint l'accès à de nouveaux médicaments contre l'hépatite $C$, coûteux mais efficaces, si bien que les caisses-maladie ne les remboursent désormais qu'aux patients atteints d'une lésion hépatique avancée.

Personne ne conteste que les dépenses de santé sont élevées en Suisse et qu'elles n'ont cessé d'augmenter au fil du temps. Mais l'analyse du système de santé devrait aussi se concentrer sur le bénéfice des prestations médicales. En effet, avoir des dépenses de santé élevées et en augmentation ne pose pas de problème en soi, tant que les soins, tels que financés, correspondent aux attentes des assurés et leur procurent un bon rapport coût-bénéfice. Pour les patients, la qualité de notre système de santé se mesure par une meilleure santé et une plus grande longévité. En conséquence, les assurés bénéficient d'un accès facile, rapide et global aux prestations de santé.

\section{Forte satisfaction des patients}

Tout ce que le système de santé apporte comme bénéfice a été relativement négligé jusqu'à présent dans le débat public. Rares sont ceux qui contestent le bénéfice qu'il génère en Suisse mais, à l'inverse du coût, ce dernier est beaucoup plus difficile à mesurer. On s'efforce de plus en plus aujourd'hui d'identifier les facteurs influant sur la notion objective ou subjective de bénéfice pour le patient, que ce soit par des évaluations de la qualité ou des enquêtes de satisfaction ${ }^{1}$.

La qualité du système de santé obtient régulièrement de bons résultats dans les sondages. D’après le Moniteur de la santé 2016 d'Interpharma, 81\% des sondés ont une opinion positive du système de santé et $75 \%$ considèrent que sa qualité est bonne à très bonne ${ }^{2}$.

Les études sur l'état de santé de la population sont également révélatrices. Par exemple, une étude de l'OCDE de $2006^{3}$ a montré que la Suisse obtenait de très bons résultats par rapport à d'autres pays de l'OCDE pour des critères objectifs et subjectifs tels que l'espérance de vie ou l'autoévaluation de l'état de santé. De plus, la variation de l'état de santé entre les différents groupes socio-économiques est moins importante en Suisse, notamment grâce à un accès généralisé de la population aux prestations de santé.

\section{Le bénéfice exprimé en francs}

Le bénéfice des prestations de santé est-il donc supérieur à leur coût? Les évaluations de la qualité, les enquêtes de satisfaction et les comparaisons internationales d'indicateurs n'apportent pas de réponse définitive à cette question pourtant centrale. Une possibilité consiste donc à quantifier les avantages en unités monétaires, pour lesquelles les économistes appliquent généralement le concept dit de la propension à payer. Mais pour l'heure, très peu d'évaluations de ce genre existent en Suisse.

Il y a près de dix ans, des chercheurs ${ }^{4}$ se sont penchés pour la première fois sur la question des avantages et du bénéfice des propositions de réforme en Suisse, sans analyser la propension à payer mais les compensations exigées, car il était principalement question de réduire le catalogue des prestations de l'assurance obligatoire, ce qui équivaut à une perte de bénéfice pour les assurés. Les résultats ont montré quelle réduction de 
prime d'assurance-maladie était nécessaire pour qu'une mesure d'économie soit acceptée.

Les pertes les plus significatives sont la restriction du libre choix du médecin et de l'hôpital: pour que les assurés acceptent une liste de médecins établie selon des critères de coût, il faudrait leur accorder une compensation de près de CHF 100 sur la prime d'assurance mensuelle (cf. fig. 1). Cette compensation serait plus ou moins divisée par deux lorsque la liste de médecins est établie non plus selon des critères de prix, mais de qualité (CHF 53) ou d'efficience (CHF 42). Elle s'élève à CHF 37 avec une limitation du choix hospitalier sans les petits hôpitaux régionaux. La perte d'avantages est également élevée si l'accès aux innovations est retardé de deux ans, par exemple lorsque de nouveaux médicaments ou implants ne sont pas inclus dans le catalogue des prestations de l'assurance obligatoire dans les deux ans qui suivent leur autorisation d'entrée sur le marché. Cette perte a été chiffrée à CHF 65 par personne et par mois. L'accès direct aux innovations a ainsi procuré un bénéfice médical aux assurés à hauteur d'environ CHF 5 milliards par an, ce qui correspond à environ 10\% des dépenses de santé de l'époque.

L'étude n'établit pas de comparaison avec les coûts.
Pourtant, il est hautement improbable d'arriver à économiser CHF 5 milliards par an par un accès retardé aux innovations. Par conséquent, une réforme de ce genre entraînerait une perte d'avantages pour les assurés plus importante que les coûts économisés.

\section{Forte propension à payer pour la fin de vie}

Une étude ${ }^{5}$ de 2016 a analysé en Suisse le niveau de propension des assurés à payer les prestations médicales en fin de vie. Comme les coûts sont particulièrement élevés pendant cette période, le rationnement des soins y est souvent requis. Cette étude montre aussi que les assurés sont prêts à dépenser beaucoup d'argent pour une meilleure santé en fin de vie: leur propension à payer s'élève à près de CHF 200000 (cf. fig. 2) pour un traitement qui prolongerait la vie d'une année supplémentaire, mais avec une excellente qualité de vie. Cette somme correspond au double du montant fixé en 2010 par le Tribunal fédéral dans son arrêt Myozyme (très remarqué à cette époque) à partir duquel le coût du traitement n'est plus approprié6. Pour les patients atteints de cancer, ce seuil de CHF 200000 pour la dernière année de vie n'est dépassé, en fonction
5 Beck, K., V. von Wyl, H. Telser et B. Fischer (2016). Kosten und Nutzen von medizinischen Behandlungen am Lebensende, Programme national de recherche PNR 67.

6 ATF 136 V 395.

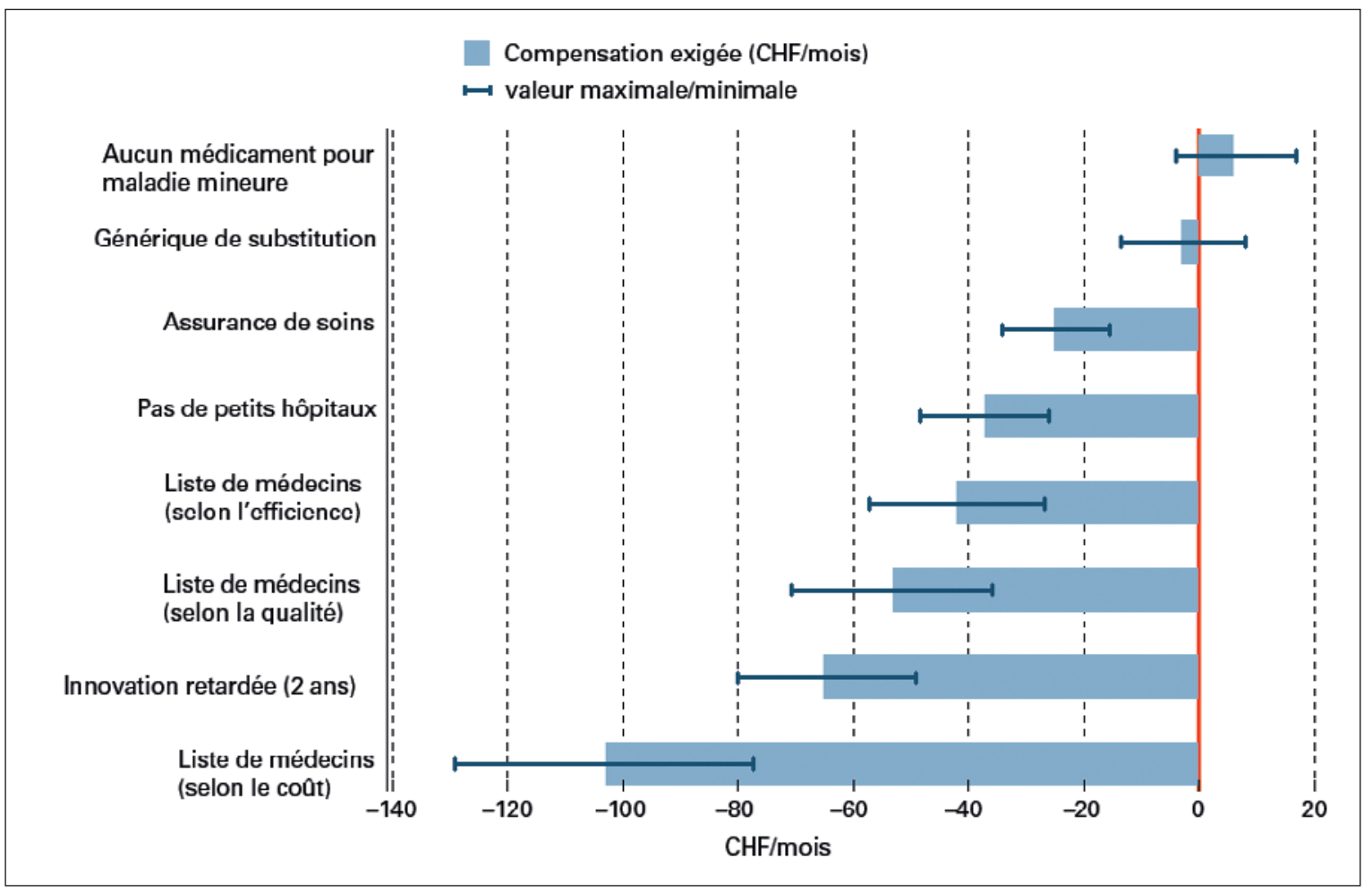

Figure 1: Perte d'avantages et de bénéfice selon la variante choisie pour la réforme, par assuré et par mois (2004). Ecart maximal selon un intervalle de confiance de $95 \%$.

La figure représente la somme à partir de laquelle un assuré moyen en Suisse accepterait volontairement une certaine restriction dans son contrat d'assurance-maladie. La plus grande perte de bénéfice (environ CHF 100 par mois) serait l'introduction d'une liste de médecins établie selon le coût. En revanche, il ne ressentirait aucune perte s'il devait payer lui-même les médicaments pour des maladies mineures: la compensation exigée est statistiquement nulle (intervalle de confiance de $95 \%$ ). 
Tableau 1.

\begin{tabular}{|c|c|c|c|}
\hline Proposition & $\begin{array}{l}\text { Valeur } \\
\text { minimale }\end{array}$ & $\begin{array}{l}\text { Valeur } \\
\text { maximale }\end{array}$ & $\begin{array}{l}\text { Compensation } \\
\text { exigée } \\
\text { (CHF/mois) }\end{array}$ \\
\hline $\begin{array}{l}\text { Liste de médecins } \\
\text { (selon le coût) }\end{array}$ & $-77,5$ & $-129,1$ & -103 \\
\hline Innovation retardée (2 ans) & $-49,2$ & $-80,1$ & -65 \\
\hline $\begin{array}{l}\text { Liste de médecins } \\
\text { (selon la qualité) }\end{array}$ & $-36,0$ & $-70,7$ & -53 \\
\hline $\begin{array}{l}\text { Liste de médecins } \\
\text { (selon I'efficience) }\end{array}$ & $-26,7$ & $-57,2$ & -42 \\
\hline Pas de petits hôpitaux & $-26,2$ & $-48,4$ & -37 \\
\hline Assurance de soins & $-15,6$ & $-34,2$ & -25 \\
\hline Générique de substitution & 8,1 & $-13,4$ & -3 \\
\hline $\begin{array}{l}\text { Aucun médicament pour } \\
\text { maladie mineure }\end{array}$ & 16,9 & $-4,0$ & 6 \\
\hline
\end{tabular}

de l'âge, que par 19\% des personnes décédées. Dans la majorité des décès, les dépenses de santé de la dernière année de vie sont nettement inférieures à ce que l'assuré moyen est prêt à payer pour cette période.

Ces études nous donnent beaucoup de raisons de penser que la plupart des patients estiment que les soins de santé sont de haute qualité, tout en étant adaptés à leurs besoins. De ce fait, les assurés sont prêts à en payer le prix. Cela ne signifie évidemment pas qu'il n'existe aucun potentiel d'économie.

Il est bien évident que le mieux serait d'économiser des coûts de santé sans s'opposer à des prestations offrant un bénéfice équivalent. Or, il existe aussi des réformes qui n'impliquent pas forcément une perte d'avantages ou de bénéfice. L'assurance pourrait par exemple ne rembourser que les génériques au lieu des préparations originales, lorsque c'est possible, et les médicaments pour les maladies mineures pourraient être à la charge des patients (cf. fig. 1). Dans tous les cas, il est important de mettre en garde contre un rationnement mis en place sur la seule perspective des coûts.

\section{Résumé}

En considérant le système de santé de notre pays du point de vue des patients et des assurés, on remarque qu'il est beaucoup question de coûts, et très peu des avantages apportés par la médecine. Cette focalisation sur les coûts risque de conduire à des erreurs d'appréciation politique avec une perte plus importante pour les assurés que les coûts économisés. La plupart des études sur le système de santé se fondent sur des indicateurs de qualité et de satisfaction, qui ne sont cependant pas suffisants pour préciser le lien entre coûts et bénéfices. Une possibilité serait donc de quantifier ces derniers en unités monétaires. Les quelques études à ce sujet en Suisse indiquent que, dans l'ensemble, les soins d'aujourd'hui génèrent davantage de bénéfices pour les assurés qu'ils ne leur en coûtent en primes d'assurance-maladie. Enfin, les assurés font état d'une forte propension à payer dans deux domaines en particulier pour lesquels un rationnement est souvent requis afin de répondre au coût élevé: l'accès direct aux innovations et les traitements en fin de vie.
Correspondance: Harry Telser Polynomics AG Baslerstrasse 44 CH-4600 Olten

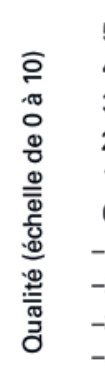

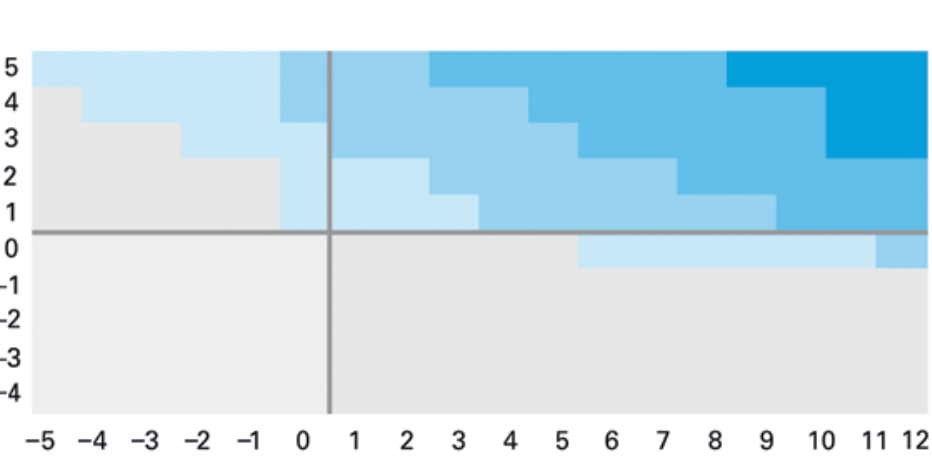

Consentement à payer $>150000 \mathrm{CHF}$

$100000-150000 \mathrm{CHF}$

50000-100000 CHF

$<50000 \mathrm{CHF}$

Aucun

\section{Durée de vie en mois}

Figure 2: Propension moyenne à payer pour une durée de vie prolongée et une meilleure qualité de vie, par patient en fin de vie (2014). Cette figure représente la propension à payer des assurés en fonction de la durée et de la qualité de vie des patients ayant encore six mois à vivre avec une qualité de vie moyenne. Le début est marqué par les lignes grises. Les valeurs négatives de la qualité indiquent une détérioration de la qualité de vie des patients, les valeurs négatives des mois matérialisent une diminution de l'espérance de vie. Les valeurs positives équivalent respectivement à une amélioration de la qualité et à un prolongement de la durée de vie. Plus la couleur d'un champ est foncée, plus la propension à payer la combinaison voulue de changement de durée et de qualité de vie est élevée. 